\title{
Significado Social de la Cerámica Nasca Temprano en el Valle de Acarí, Perú
}

\section{Social Significance of the early Nasca Ceramics in the Acari Valley, Peru}

Lidio M. VALDEZ

\section{Resumen}

En este trabajo, mi principal objetivo es evaluar el significado social de la cerámica Nasca temprano proveniente del valle de Acarí, donde se han recuperado muestras de alfarería Nasca temprano. Hasta hace poco, dichas muestras sirvieron para sostener que un estado Nasca temprano centrado en Cahuachi habría sido la fuerza principal que introdujo la cerámica Nasca hacia los valles vecinos, siendo uno de estos Acari. Lejos de confirmar dicha propuesta, los especialistas mantienen un consenso casi unánime en sostener que Nasca nunca llegó a constituir un estado; por su lado, los recientes estudios realizados en el valle de Acari dan a conocer que el estilo Nasca nunca sustituyó al estilo local. La introducción de la fina alfarería Nasca al valle de Acari parece estar asociada con personajes de poder, quienes utilizaron objetos exóticos para expandir y consolidar sus prestigios personales. Ejemplares de la cerámica Nasca, si bien presentes en Acari, siguen siendo limitados.

Palabras Clave: Andes Centrales, Costa Sur, Periodo Intermedio Temprano, Nasca.

Department of Archaeology, University of Calgary. E-mail: lidio9@yahoo.es Recibido: Octubre 2007. Aceptado: Marzo 2008. 


\begin{abstract}
The main purpose of this paper is to assess the social significance of the early Nasca ceramics found in the Acari Valley, of the South Coast of Peru. Until recently, Nasca ceramics found in Acari have been used to argue that an early Nasca state centered at Cahuachi invaded Acari, resulting in the eventual introduction of Nasca artifacts. Far from confirming this speculation, scholars agree that Nasca never established a centralized political authority, while recent studies in Acari show that Nasca ceramics never substituted the local pottery tradition. The introduction of the polychrome Nasca ceramics to the Acari Valley appear to be associated with the emergence of local elites who used foreign exotic goods to enhance personal prestige. Samples of Nasca ceramics are found in Acari, but are limited and never replaced the local style.
\end{abstract}

Key Words: Central Andes, South Coast, Early Intermediate Period, Nasca

\title{
INTRODUCCIÓN
}

De los varios materiales culturales recuperados de los sitios arqueológicos, la cerámica es el artefacto que ha recibido mayor atención de los especialistas, especialmente en regiones como los Andes Centrales. Este hecho obedece, como advirtió Lanning (1967:24), a varios factores, los mismos que varían desde su abundancia y fácil preservación en los sitios arqueológicos, hasta los notables y rápidos cambios de orden estilístico, útiles para determinar la cronología relativa de los sitios arqueológicos (Sackett 1985:277). Por cuanto los cambios estilísticos ocurren de un espacio geográfico a otro (espacio) y de un tiempo a otro (tiempo), dichas variaciones son de vital importancia en el análisis arqueológico. En efecto, en base a análisis estilísticos de la cerámica, Max Uhle fue hábil en aislar las varias tradiciones culturales de los Andes Centrales y establecer la inicial secuencia cronológica (Strong 1948:94). Dicha secuencia maestra fue posteriormente adoptada por Kroeber (1927, 1944), y sirvió para definir la categoría de "horizontes" (Kroeber 1944:108; Strong 1948:94; Willey 1948:8). La secuencia cronológica en vigencia (Rowe 1960), edificada sobre la base de los trabajos anteriores, fue también elaborada en base a las diferencias estilísticas.

Así como en otros lugares (ver, Dunnell 1986:32; Conkey 1990:8; Plog 1990:61-62), la inicial identificación de las diferentes tradiciones cul- 
turales de los Andes Centrales (i.e., Vicús, Moche, Recuay, Lima, Nasca, Huarpa) fue basada estrictamente en las diferencias estilísticas de la cerámica. En efecto, al clasificar el material arqueológico (cerámica) en términos de estilo, los especialistas no tardaron en notar los contrastes estilísticos entre una cultura y otra (Bennett y Bird 1949:115; Lanning 1967:122). Por su parte, la homogeneidad estilística de una cultura, si bien no del todo ignorada, sólo fue esbozada en términos de continuidad poblacional, aislamiento y la aparente poca comunicación entre una cultura y otra (Bennett y Bird 1949:69, 115). Sin embargo, y siempre enfatizando el aspecto cronológico, los estilos o tradiciones culturales fueron divididos en varias fases que denotaban el desarrollo gradual de una determinada cultura, desde luego siguiendo un razonamiento evolucionista. El mejor ejemplo de estudios de esta naturaleza lo encontramos en el trabajo de Menzel, Rowe y Dawson (1964). Este notable interés de varias generaciones de arqueólogos fue parte, y consecuencia, de toda una corriente arqueológica que caracterizó a la América en general (ver Conkey 1990), y cuyo mayor objetivo fue establecer secuencias cronológicas y culturales, como un paso necesario para explicar el proceso histórico-cultural (Willey y Sabloff 1980:97).

Al mismo tiempo, la identificación de los mayores centros culturales y las mismas tradiciones culturales se basó en la recurrencia estilística (Bennett y Bird 1949:68-69). De esta manera, y así como Donald Proulx expuso sucintamente, en los Andes Centrales una cultura está básicamente definida en base a la cerámica, y cuando el mismo estilo de cerámica ocurre en dos o más sitios se asume directamente que sus habitantes pertenecieron a la misma cultura (Proulx 1968:99). Cabe recordar que hasta hace poco, las muestras de cerámica utilizadas para tales objetivos fueron las piezas decoradas. Por lo tanto, para los especialistas la cerámica siempre fue, y continúa siendo, un instrumento diagnóstico de mucha importancia (Lumbreras 1984:3).

En este trabajo, es mi intención ubicar el caso del periodo Intermedio Temprano (circa 1 - 550 d.C.) del valle de Acarí, prestando particular atención a la alfarería Nasca temprano proveniente de dicho valle. Para su efecto, primero se ubica el motivo de estudio dentro del contexto arriba referido, para luego enfocar a la parte simbólica de los artefactos. En particular, considero de mucha utilidad prestar atención al origen y lugar de proveniencia de los artefactos. Efectivamente, a parte de su función cotidiana, los objetos son símbolos que ponen en contacto dos o más sociedades diferentes. Como símbolos de origen foráneo, el valor de los objetos ya no radica en la función que debieron cumplir, o para la cual fueron manufacturados, sino está de- 
terminada en la conexión con lugares específicos, que para muchos no dejan de ser lugares exóticos, cuyos nombres y existencia sólo se conocen a través de segundas o terceras personas.

En el contexto de la arqueología Andina, existen muchos lugares de importancia, pero de cuya existencia muchos individuos probablemente sólo se enteraron por intermedio de otras personas que tuvieron la oportunidad y privilegio de llegar hasta dichos centros. Cuzco, como capital de un imperio nunca antes visto en el contexto Andino, fue uno de aquellos centros. Pachakamaq, Wari, Tiwanaku, Chavín de Huántar, fueron otros lugares que incluso muchos años después de su inicial abandono siguieron manteniendo su prestigio (ver Burger 1992: 265). Para la costa sur, no encontramos sitios de similar prestigio y poder que los anteriormente mencionados; una excepción es Cahuachi, el centro ceremonial Nasca temprano ubicado en el valle de Nasca (Silverman 1993; Silverman \& Proulx 2002; Valdez 1994). Estudios efectuados en este sitio revelan que Cahuachi fue también el centro donde una mayor cantidad de la fina alfarería Nasca fue utilizada (Silverman 1988).

\section{LA COSTA SUR}

Con anterioridad a la identificación de Cahuachi como centro ceremonial, éste fue visualizado como una ciudad urbana y capital del floreciente estado Nasca temprano (Rowe 1963). Dentro del marco de esta interpretación, la presencia de la fina y policroma cerámica Nasca temprano fuera de los valles de la cuenca del Río Grande e Ica fue interpretada como una manifestación de la expansión militarista de Nasca (Lanning 1967; Proulx 1968; Massey 1986). La presencia de los así llamados "cabezas trofeo" y de sitios al parecer "fortificados" parecía reforzar la hipótesis que interpretó a Nasca como un estado militarista y expansivo.

Un rasgo característico de los sitios del periodo Intermedio Temprano del valle de Acarí es la presencia de grandes muros que encierran su perímetro (Valdez 2000:162), los mismos que fueron inicialmente identificados por Rowe (1963:11-12) como "fortificaciones". Siguiendo dicha sugerencia, otros investigadores enfatizaron la aparente función defensiva de los muros perimétricos de los sitios de Acarí. Por ejemplo, Lanning (1967:121) no sólo enfatizó que Acarí fue conquistado por Nasca, sino también dio a entender que a lo largo de cada valle de la costa sur existen numerosos sitios fortificados pertenecientes al periodo Intermedio Temprano. Además, Lanning da referencia a la presencia de armas de guerra, llevando a la obvia conclusión 
que este periodo fue convulsionado. Del mismo modo, Proulx (1968:97) vio a Nasca (circa 1 - 550 d.C.) como una cultura expansiva y cuya influencia fue sentida en el valle de Acarí. Proulx (1971:20) analizó los así llamados "cabezas trofeo" y concluyó que éstas eran producto de la guerra. Partiendo de esta misma línea de análisis, Massey (1986:338) identificó a Nasca como un estado que administró varios valles de la costa sur desde su centro establecido en Cahuachi. Dentro de esta perspectiva, los objetos Nasca hallados en Acarí fueron interpretados como directo producto de esta invasión y conquista Nasca.

Las últimas tres décadas fueron fructíferas para el estudio de la cultura Nasca. Efectivamente, varios estudios fueron llevados adelante, muchos de los cuales orientados a verificar planteamientos que hasta entonces aparecían como conclusiones definitivas (Carmichael 1988, 1995; Silverman 1993; Silverman y Proulx 2002; Valdez 1998; Vaughn 2000). Fue entonces que las viejas conjeturas, formuladas a partir de evidencias superficiales, no pasaron el escrutinio hecho mediante excavaciones arqueológicas, y fue así que Nasca empezó a ser visualizado de una manera distinta (Silverman 1993, 2002; Silverman y Proulx 2002). La mejor síntesis de esta etapa de transición en los estudios Nasca, la ofrece Proulx (2001:129) al sostener:

"Bajo el viejo modelo que visualizó a Nasca como un estado primitivo con un gobierno central que emanó desde su capital Cabuachi, fue fácil sostener que los Nasca se expandieron mediante medios militares, sometiendo los valles vecinos e imponiendo su cultura a sus habitantes..."

Es precisamente dentro de este contexto interpretativo que se necesita explicar el significado social de la cerámica policroma Nasca proveniente del valle de Acarí (Figura 1). Para poner en perspectiva la presente discusión, primero se da referencia a los recientes trabajos arqueológicos efectuados en el valle de Acarí. Dicha información no sólo es básica, sino también permite discutir el carácter de los contextos excavados en sitios específicos. Es de dichos contextos que se extrae la información que permite abordar el tema motivo de esta contribución. 


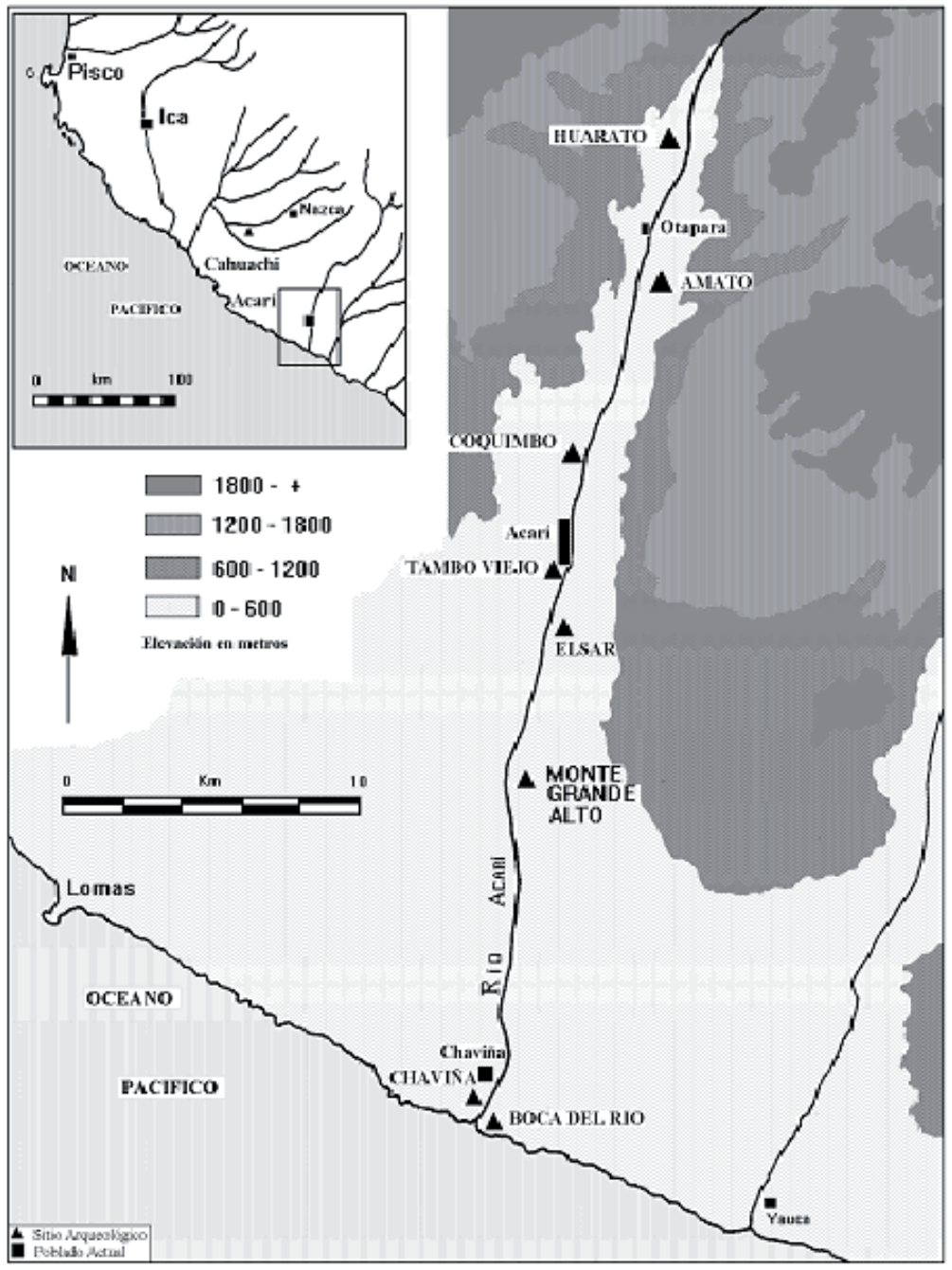

Figura 1. Ubicación de los sitios del periodo Intermedio Temprano del valle de Acarí.

Figure 1. Location of the Early Intermediate Period sites of the Acari Valley

\section{El PROYeCto ARQUeOLÓGico AcARí}

“.. catalogamos 25 sitios en todo el valle de Acari entre el gran cementerio Nazca 2-3 de Huarato a la cabeza del valle y los basurales de moluscos de Chaviña en la boca del rio" (Rowe 1956:140).

"Más arriba en el valle, en Chocavento, hay otro sitio de habitación fortificado, muy parecido a Tambo Viejo aunque su tamaño es mucho más pequeño. Todavia más arriba existen dos sitios adicio- 
nales de características parecidas, uno es en Amato y otro en Huarato. Ambos son más pequeños que Chocavento. Todos estos sitios parecen haber sido construidos y abandonados durante el Periodo Intermedio Temprano fase 3" (Rowe 1963:11-12).

En un esfuerzo por esclarecer aspectos de la arqueología de Acarí que permanecen inciertos, durante estos últimos años se ha logrado efectuar trabajos de excavación en los sitios de Huarato y Amato (Valdez 2007, 2007). Dichos estudios vienen siendo efectuados dentro del programa de investigaciones del "Proyecto Arqueológico Acarì" dirigido por el autor. Inicialmente, Huarato y Amato fueron identificados por Rowe (1963) como sitios pertenecientes al periodo Intermedio Temprano, pero en ninguno de estos sitios se habían efectuado trabajos de excavación. En consecuencia, la excavación realizada primero en Huarato (Valdez 2005a, 2005b, 2005c, 2007) y luego en Amato constituyen logros largamente esperados.

Entre otros, los trabajos arriba mencionados, y otros a proseguir en las temporadas que siguen, están orientados a explicar mejor la situación de este valle durante el referido periodo. Por cuanto este fue el tiempo durante el cual la cultura Nasca floreció en los valles de Ica y la cuenca del Río Grande, no se puede ignorar el caso Nasca, y de manera muy particular la influencia del centro ceremonial Nasca temprano de Cahuachi (Silverman 1993; Valdez 1994, 1998). Por esta razón, determinar la naturaleza de las relaciones entre Nasca y Acarí es uno de los focos principales de este proyecto. Otros aspectos que se busca definir están relacionados a subsistencia, uso del espacio, especialización, construcción y los patrones mortuorios.

En primer lugar, Huarato (Figura 2) es un sitio que presenta una ocupación perteneciente a las primeras fases del Periodo Intermedio Temprano (circa 1 - 400 d.C.) y está ubicado en la parte superior media del valle. Como muchos otros, este ha sido objeto de intensas actividades clandestinas del huaqueo, pero nunca excavado sistemáticamente. Una excepción fue la intervención de Carpio (1942), pero que cuyos resultados nunca fueron bien sustentados. Además del huaqueo, otras actividades contemporáneas, como la ampliación de los campos de cultivo, han contribuido al deterioro físico del sitio arqueológico. Como resultado, la extensión original del sitio ha sido considerablemente reducida, mientras lo que se conoce del sitio sigue siendo muy limitado. En efecto, hasta hace poco la única referencia acerca del sitio fue la breve mención de Rowe $(1956,1963)$, la misma que no deja de ser muy general. 


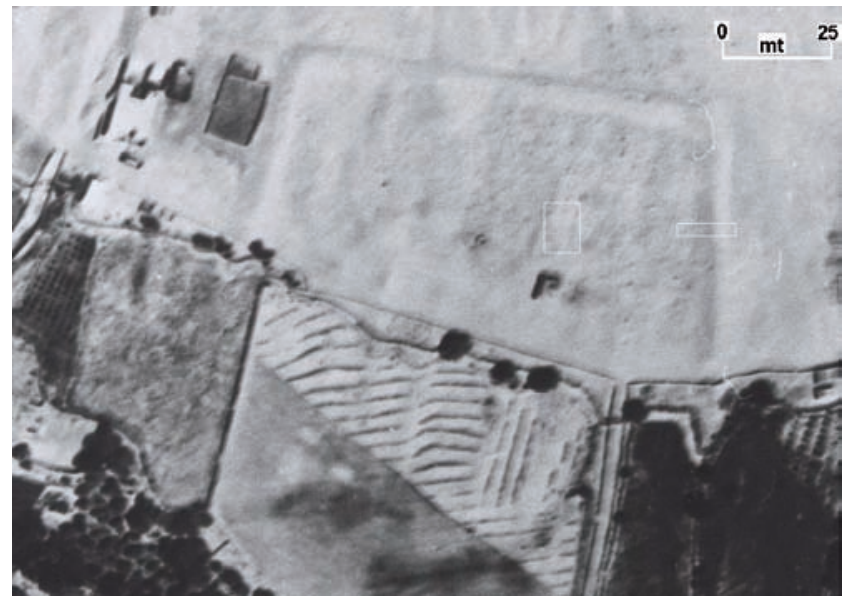

Figura 2. Foto aérea del sitio de Huarato con indicación de los sectores excavados.

Figure 2. Aereal view of Huarato with indication of the excavated sectors

De acuerdo a Carpio (1942:488), así como Lothrop y Mahler (1957:3) y Proulx (1989:73), es posible que Julio C. Tello haya llegado hasta Huarato. De acuerdo a dicha versión, Tello habría sido uno de los primeros en lamentar la destrucción de los sitios como resultado del saqueo. Posteriormente, Rowe (1956:140) describió Huarato como "un gran cementerio Nazca 23", probablemente en base a la cantidad de huesos humanos observados en la superficie. Desafortunadamente, el pillaje continúa y pone en peligro la misma existencia del sitio.

En segundo lugar, durante las temporadas de 2005 y 2006 los trabajos se centraron en el sitio de Amato (Figura 3). A diferencia de Huarato, Amato nunca fue excavado aunque sí intervenido por los huaqueros. Sin embargo, dichas intervenciones (excepto el cementerio del lado NE y que pertenece a periodos posteriores) no han sido intensas como en Huarato. Por lo tanto, Amato se encuentra en buen estado de conservación. El sitio aparece del todo encerrado por un muro largo perimétrico, aspecto este que otros sitios contemporáneos de este valle lo comparten. Al interior de dicha construcción son visibles muchas otras estructuras y muros largos que dividen el sitio en varias partes. 


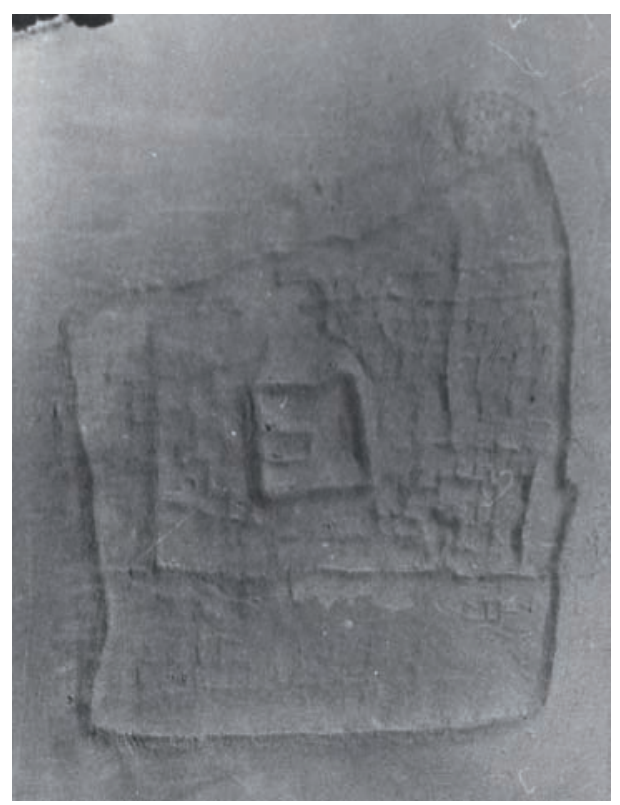

Figura 3. Foto aérea del sitio de Amato con indicación del sector central.

Figure 3. Aereal view of the Amato site with indication of the central sector

\section{La Evidencia Arqueológica}

"El valle de Acarí fue el último de la costa sur en mantener una orientación y asociación predominantemente norteña" (Menzel y Riddell 1986:105).

Mientras queda evidente que apenas se ha dado inicio en los estudios sistemáticos en los sitios de Acarí, los resultados hasta hoy disponibles ya presentan un escenario distinto de lo que se aceptó hasta hace poco. El caso específico es con respecto a las formas de enterramiento de Acarí (Valdez 2005a, 2005b, 2006, 2007) que hasta hace poco eran desconocidos. Por lo tanto, y por primera vez, estas nuevas evidencias provenientes de Acarí pueden ser comparadas y contrastadas a los patrones de enterramientos Nasca (Carmichael 1988). De particular importancia es notar que en la opinión de Carmichael (1988:306; Orefici y Drusini 2003:118), la policroma cerámica Nasca es un elemento que siempre está presente en los entierros Nasca.

Las formas de enterramiento de Acarí han sido discutidos en otros trabajos (Valdez 2005a, 2006), razón por la cual aquí sólo se hace una breve referencia. Teniendo en cuenta las variaciones en cuanto a la construcción de las tumbas se refiere, ya se pueden distinguir hasta cuatro formas de enterramientos para los sitios de Acarí. La primera consiste de un simple hoyo 
excavado directamente en el suelo natural y en donde el cadáver había sido depositado. Entierros de este tipo no contienen ofrenda alguna. La segunda forma de enterramiento es en urnas, y con la excepción de una excavada recientemente en Amato, todas pertenecen a individuos infantes (Valdez 2006:7). Un total de 6 entierros intactos (y 5 saqueados) en urnas fueron excavados a la fecha, pero ningunos poseían ofrendas de cerámica. Una excepción viene a ser un entierro excavado en 2004 en Monte Grande Alto y que poseía cerca de la cabeza una pequeña vasija cerrada, decorada en el estilo local. La tercera forma de enterramiento consiste de hoyos también excavados directamente en el suelo natural que -a diferencia de la primera forma- disponen de techos de caña o de lajas, y en cuyo interior fueron depositados los cuerpos (Valdez 2005a:50, 2006:8). Este tipo de enterramientos incluyen adultos e infantes, y con la excepción de una tumba excavada en Huarato (Valdez 2005a), la cerámica no es un elemento que recurre en dichos contextos. La cerámica encontrada en la tumba de Huarato es monocroma y de acabado simple (Valdez 2006: Figura 13) y definitivamente no es Nasca. Finalmente, durante la temporada de 2004 una estructura conteniendo el resto de tres individuos adultos fue excavada en Tambo Viejo (Valdez 2006: Figura 8). La estructura había sido saqueada en parte, pero dos de los entierros, ambos separados del primero por un pequeño muro, aún estaban en sus contextos originales. Como parte del ajuar funerario de uno de los individuos, uno de sexo femenino, se halló una pata trasera de camélido. El siguiente individuo encontrado in situ no poseía ofrenda alguna. En el relleno depositado sobre este se encontraron fragmentos de cerámica del estilo local (Valdez 2006:11).

Recientemente, en la parte central del sitio de Amato se llegó a excavar varias decenas de esqueletos humanos con indiscutibles evidencias de haber sido decapitados. Lo interesante para esta discusión es la presencia de un entierro perteneciente a un individuo adulto de sexo masculino asociado a los cuerpos decapitados y que sí mantenía su cabeza. Dicho individuo, cuya edad oscila alrededor de los 60 años, poseía una pechera hecha de cuentas y cientos de huesos de alguna ave pequeña. Al igual que los entierros encontrados en Huarato (Valdez 2005a), este individuo había sido depositado manteniendo una posición sentada, con las rodillas flexionadas hacia el pecho, las manos cruzadas a la altura de los pies, y orientado hacia el norte. Además de los individuos decapitados, también se hallaron un total de cuatro camélidos jóvenes, colocados como ofrendas cerca del individuo adulto. Finalmente, cerca de los camélidos fueron hallados varios ejemplares de Spondylus trabajados. Otros objetos encontrados en asociación al individuo incluyen las vainas del maní y un mate que había sido depositado 
precisamente cerca de los pies. Lo sobresaliente de todo este hallazgo, y los anteriormente referidos, es la ausencia de la policroma cerámica Nasca. Tal como se anotó líneas adelante, la cerámica Nasca es un objeto recurrente en los entierros Nasca.

El hallazgo de las piezas de Spondylus en Amato es clara prueba de la importancia de los productos exóticos en este valle, los mismos parecen haber estado conectados con individuos de prestigio. Obviamente el individuo adulto de Amato era uno de dichos personajes y su importancia se puede evaluar observando el número de personas sacrificadas y la presencia de productos exóticos. Un fechado de carbón obtenido para dichos contextos arrojó una fecha de 15 d.C., y sugiere que este contexto antecede al periodo de la aceptación en Acarí de la policroma cerámica Nasca como objeto de lujo.

Además de los entierros, tanto en Huarato como en Amato se llegó a excavar otros contextos que contenía desechos y otras evidencias domésticas. En ambos casos, sin embargo, la presencia de fragmentos de la cerámica Nasca es raro. Por ejemplo, en el sector 2 de Huarato se llegó a definir recintos identificables como criaderos de cuyes y silos de almacenamiento, además de otros recintos. En asociación a todas estas estructuras, y otras similares, la cerámica Nasca simplemente no ocurre.

En las recientes excavaciones realizadas en Amato también se logró exponer contextos con deposiciones que contienen restos domésticos. Este es el caso concreto de la esquina sur-oeste del recinto central donde se hallaron los cuerpos decapitados. Allí se definió una deposición profunda conteniendo un acumulamiento de desechos de carácter doméstico y como tales conteniendo la mejor colección de cerámica para un sitio del periodo Intermedio Temprano de Acarí. Además de la cerámica, otros restos que ocurren en el referido contexto son restos de plantas y animales, ceniza, tejidos, huesos trabajados, y muchos otros restos orgánicos. Sin embargo, fragmentos Nasca simplemente no ocurren en dicho contexto. Resumiendo, los trabajos hasta hoy realizados en Acarí no han resultado en el hallazgo de contextos con alfarería Nasca temprano. Una rara excepción viene a ser una deposición excavada al lado exterior del muro norte de Huarato, donde sÍ ocurren los fragmentos Nasca temprano.

\section{La Cerámica Nasca Temprano en Acarí}

Hasta antes de las excavaciones efectuadas en Huarato y Amato existía la tendencia a asumir que dichos sitios estaban asociados con la cerámica Nasca temprano. Aunque ya se había cuestionado dicha posibilidad (Valdez 
1998), esta persistía en particular de parte de quienes aún consideran Nasca como un estado. Otros investigadores, incluido Silverman y Proulx (2002; Proulx 2001), ya no consideran Nasca como un estado.

Previamente, se había sugerido que tal vez determinados individuos de Acarí utilizaron objetos exóticos, incluida la bella cerámica Nasca, como bienes de lujo que simbolizaron status y prestigio (Valdez 1998). Con este modelo se esperaba que como objetos que connotaban prestigio y status, la ocurrencia de la cerámica Nasca formará parte del ajuar funerario de determinados individuos (Valdez 1998:162, 183). Sin embargo, y contrario a las expectativas, ningún entierro hasta hoy excavado presenta la fina y policroma cerámica Nasca.

La ausencia de entierros conteniendo objetos Nasca no está del todo descartado y el modelo en mención no está del todo descartado. Considerando el fechado obtenido para Amato, queda obvio que los eventos observados con los trabajos en Acarí pertenecen a las fases iniciales del desarrollo de los sitios del periodo Intermedio Temprano de Acarí. Al mismo tiempo, es oportuno recordar que el auge del centro ceremonial Nasca de Cahuachi no se dio hasta aproximadamente los años 200 o 250 de la presente era. En consecuencia, la llegada de las primeras muestras de objetos Nasca al valle de Acarí debe estar relacionada al prestigio de Cahuachi. En definitiva existen muestras de cerámica Nasca y otros objetos Nasca en Acarí (Valdez 2006: Figura 14), pero estas parecen haber llegado mucho tiempo después del establecimiento de sitios como Huarato y Amato. Esta interpretación deja abierta la posibilidad de que la presencia de los objetos Nasca en este valle no guarde ninguna relación con los muros perimétricos de los sitios de Acarí y como tales dichos muros parecen no haber sido edificados para resistir la posible ofensiva militar Nasca.

En base a las nuevas evidencias, se hace evidente que antes y durante el tiempo que las primeras muestras de cerámica Nasca temprano fueron introducidos hacia Acarí, en este valle existía un estilo local (Valdez 1998, 2000, 2007). Artística y tecnológicamente, dicha cerámica es de acabado simple en comparación a la policroma cerámica Nasca temprano. Por ejemplo, las vajillas en el estilo local son de superficie áspera, sin engobe, y las decoraciones consisten de motivos simples ejecutados sin mayor cuidado (Figuras 4 y 5 ). Estos definitivamente no son comparables a los diseños Nasca. 


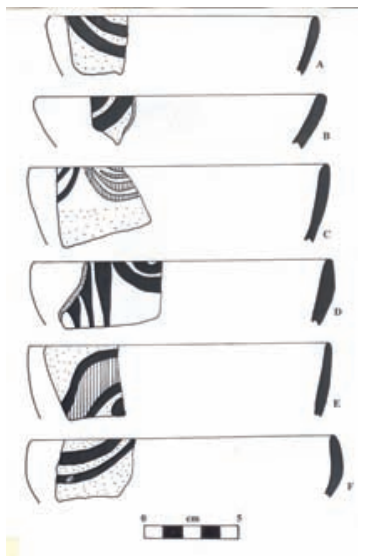

Figura 4. Cerámica local proveniente de Huarato

Figure 4. Local ceramic sherds from Huarato

No cabe duda que la cerámica Nasca temprano llegó hasta el valle de Acarí (Figura 6). Aunque queda obvio que mayores trabajos se hacen necesarios para definir el contexto de la cerámica Nasca en Acarí, parece probable que la población Nasca no participó en la introducción de la cerámica Nasca al valle de Acarí. En su lugar, los responsables debieron haber sido los personajes que buscaron agrandar sus prestigios y donde la posesión de objetos de lujo y objetos foráneos fue de vital importancia. En definitiva, la cerámica Nasca proveniente de Acarí fue manufacturada fuera de este valle (Valdez 1998). Finalmente, y no obstante su aceptación en Acarí, el estilo Nasca nunca logró sustituir a la cerámica local. Esto podría estar explicando su limitada distribución como producto de su asociación a individuos de prestigio, quienes obviamente debieron haber sido también pocos.

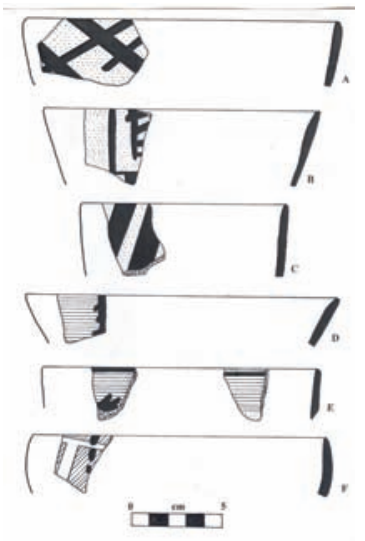

Figura 5. Cerámica local proveniente del recinto central de Amato

Figure 5. Local ceramic sherds from the central enclosure of Amato 
En consecuencia, y aparte de Tambo Viejo, Coquimbo y Boca del Río donde todavía no se ha excavado, el único sitio donde hasta la fecha se ha recuperado una buena colección de cerámica Nasca viene a ser Huarato. Sin embargo, incluso en el referido sitio los fragmentos Nasca fueron hallados en las afueras del muro perimétrico. Dicha deposición, fuera del sitio, podría estar indicando la proveniencia foránea de dichos artefactos e incluso su posible rechazo por parte del resto de la población local. Por el momento, la mayor presencia de la cerámica Nasca temprano en Huarato se puede empezar a explicar en términos del acercamiento físico del sitio al territorio Nasca. Efectivamente, Huarato es el sitio de todo el valle de Acarí más próximo a cualquier asentamiento Nasca, especialmente al área de Las Trancas. Esta observación gana mayor sentido si se toma en consideración la presencia de asentamientos Nasca temprano en dicha zona (Schreiber 1999:168). Como tal, no está por demás sugerir que dicho acercamiento tal vez fue una de las razones que convirtieron a Huarato en una suerte de puerta de ingreso de los objetos Nasca hacia el valle de Acarí.

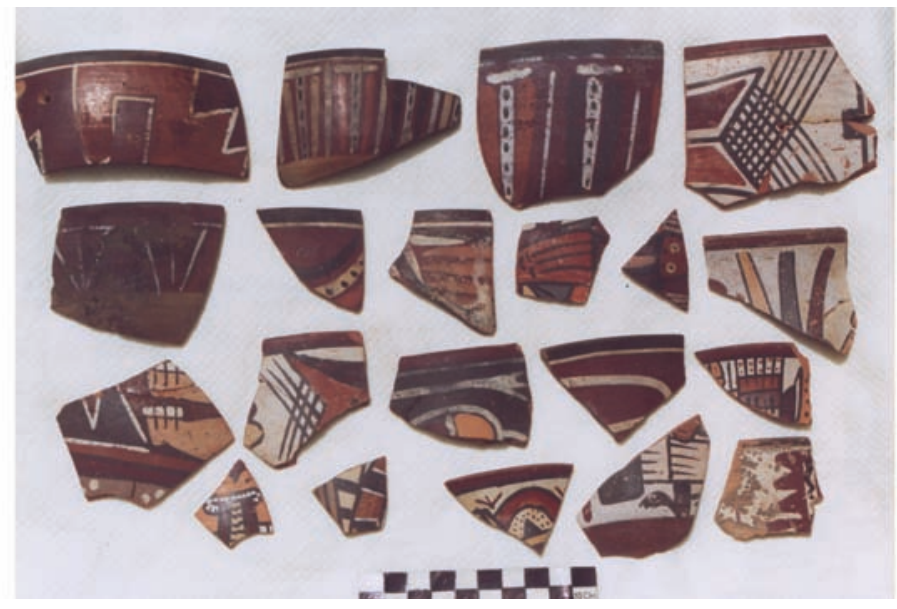

Figura 6. Cerámica Nasca temprano proveniente del lado exterior del muro Norte de Huarato.

Figure 6. Early Nasca ceramic sherds found outside the northern wall of Huarato

Se puede anticipar, además, que dicho acercamiento haya permitido una mayor fluidez en las relaciones sociales entre los habitantes de Huarato y los del valle de Las Trancas. Dicha fluidez pudo haber resultado no sólo en el intercambio de objetos, sino sobre todo en matrimonios, permitiendo de este modo la reubicación de determinadas personas en lugares distintos. En consecuencia, esta es una posibilidad alterna que la investigación arqueológica debe 
considerar con mayor seriedad para así determinar si en el pasado se dieron reubicaciones poblacionales.

A parte de la misma cerámica Nasca, ya existen evidencias que señalan que otros productos Nasca también hicieron su ingreso hacia Acarí, cumpliendo probablemente una función idéntica a la de la cerámica. El primer caso concreto se trata del mate pirograbado (Figura 7) encontrado en la superficie del sitio de Monte Grande Alto (Valdez 2000). Otro objeto viene a ser una diadema de oro laminado y decorado en el estilo Nasca recuperado del sitio de Huarato (Figura 8). La presencia de este objeto en particular abre la posibilidad de que determinados personajes residentes de Huarato adquirieron status especiales en virtud de su conexión con Nasca y los objetos Nasca. Por lo tanto, los objetos Nasca sí llegaron hasta Acarí, pero al parecer llevados por la misma gente de Acarí. El objetivo parece haber sido elevar el status social de personajes específicos. Si este fue el caso, en este proceso de la introducción de los objetos Nasca al valle de Acarí la población Nasca tal vez nunca participó. Cualquiera haya sido el mecanismo que resultó en la introducción de los artefactos Nasca, no hay duda que tales objetos fueron introducidos a un contexto de una tradición local (Figura 9).

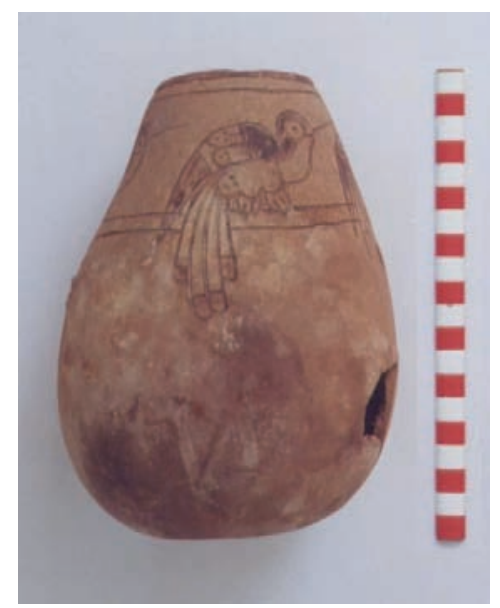

Figura 7. Mate decorado en el estilo Nasca temprano proveniente de Monte Grande Alto.

Figure 7. Decorated gourd with early Nasca designs from Monte Grande Alto

Teniendo en consideración los recientes hallazgos, todo parece indicar que las evidencias hasta hoy recuperadas mediante las excavaciones efectuadas pertenecen a las fases iniciales del desarrollo local y cuando la interacción entre Huarato y Nasca fue aún muy limitada. Los fechados de carbón 14 que iremos obteniendo en el curso de los trabajos verificarán nuestros plan- 
teamientos. Esto desde ya confirma que existe una tradición local en este valle y que fue efectivamente al contexto de dicha tradición local que fueron introducidos los objetos Nasca.

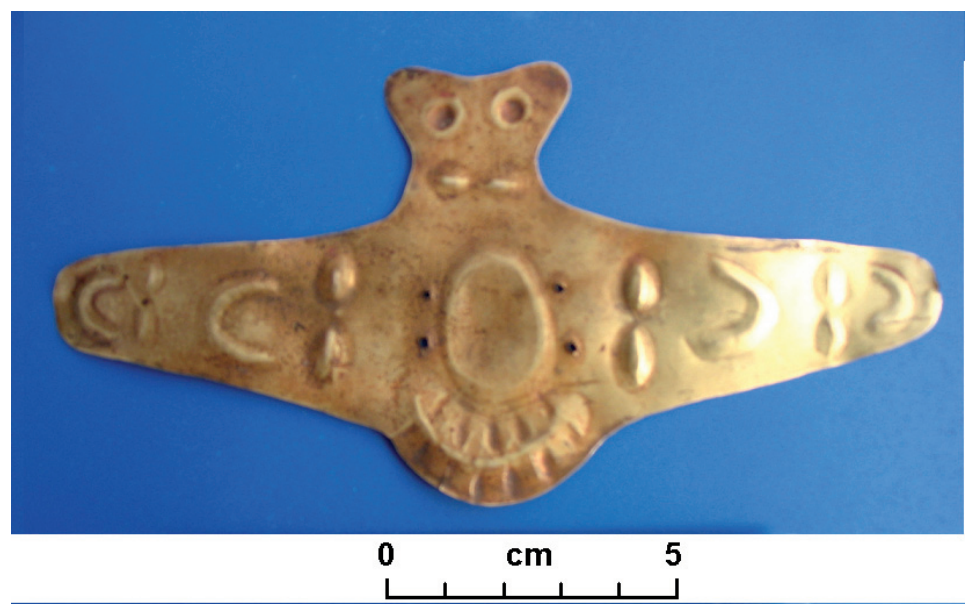

Figura 8. Diadema de oro en el estilo Nasca temprano proveniente de Huarato.

Figure 8. Head ornament made of gold in the early Nasca style from Huarato

Por su parte, merece anotar que los trabajos que se vienen efectuando en Acarí dan a conocer que entre Nasca y Acarí existen muchos elementos comunes. Esta no debe extrañar considerando la cercanía geográfica entre ambos valles. A parte de las diferencias en cuanto a la forma de los asentamientos y los estilos de cerámica se refiere, otros elementos y prácticas parecen haber sido generalizadas a lo largo de toda la Costa Sur. Esta incluye el uso de los adobes cónicos, la elaboración de las antaras de arcilla (aunque el acabado es menos sofisticado en Acarí), la obtención de las "cabezas trofeo", y la utilización de una serie de plantas domésticas. Un análisis más detallado, el que se espera efectuar en la medida del progreso de los trabajos de campo, probablemente revelará variaciones que por ahora no parecen ser obvias. 


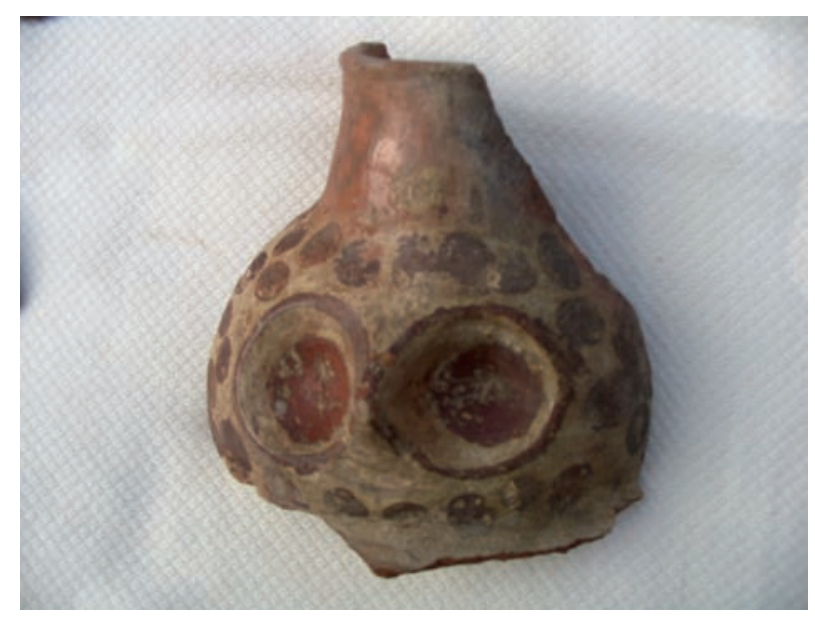

Figura 9. Cerámica local proveniente del recinto central de Amato.

Figure 9. Local ceramic sherds from the central enclosure of Amato.

En síntesis, la función de objetos determinados, como la misma cerámica, va más allá de aquella para la cual el artefacto fue originalmente manufacturado. Por su asociación a lugares y personajes de prestigio, un cuenco de cerámica puede ser fácil e intencionalmente transformado, de ser un utensilio de uso cotidiano a otro que simboliza prestigio y status. Siguiendo el modelo que visualizó Nasca como un estado expansivo, se esperaría encontrar una mayor presencia de elementos Nasca en Acarí, y en algunos sitios incluso la sustitución total del estilo local. Sin embargo, ninguno de estos escenarios es visible a la fecha. En lugar de la conquista, el nuevo modelo explica mejor los mecanismos que en última instancia llevaron a objetos determinados fuera de su contexto inicial. Dicho mecanismo fue evidentemente social, donde determinados individuos parecen haber participado activamente en el proceso de adquisición de artefactos capaces de transformar status sociales existentes.

Agradecimientos: Expreso mi gratitud y agradecimiento al Instituto Nacional de Cultura por los permisos cedidos para poder llevar adelante los trabajos de investigación en el valle de Acarí. Mis gracias también para los organizadores del IV Seminario de Arqueología UNFV por haber facilitado mi participación en dicho evento. Todo intento de trabajo hubiera sido simplemente imposible sin la participación de los integrantes del "Proyecto Arqueológico Aca$r i$, cuya lista sería larga y tal vez incompleta para enumerarlos. Del mismo modo, mi profundo reconocimiento para Ángel Iglesias y esposa, Rosa Mazuelo, por su permanente apoyo incondicional durante las varias temporadas de campo. Finalmente, mi agradecimiento para mi esposa Katrina Bettcher, y mis hijos Kai y Kía, por el amor y cariño que siempre me brindan. 


\section{BiBLIOGRAFÍA}

Bennett, W. y J. Bird. 1949. Andean Culture History: the archaeology of the Central Andes from early man to the Incas. The Natural History Press, Garden City, New York.

Burger, R. 1992. "The Sacred center of Chavín de Huántar". En The Ancient Americas: Art from Sacred Landscapes, editado por R. F. Townsend, pp. 265-277. The Art Institute of Chicago, Chicago.

Carmichael, P. 1988. Nasca Mortuary Customs: Death and Ancient Society on the South Coast of Peru. Ph. D. Dissertation, Department of Archaeology. University of Calgary, Calgary.

Carmichael, P. 1995. "Nasca burial patterns: social structure and mortuary ideology". En Tombs for the Living: Andean Mortuary Practices, editado por T. D. Dillehay, pp: 161-187. Dumbarton Oaks, Washington D.C.

Carpio, A. 1942. "Datos sobre la arqueología de los valles de Acarí y Duaca". XXVII Congreso Internacional de Americanistas, Actas y Trabajos Cientificos de la Segunda Sesión, Vol. I: 435-529. Lima.

Conkey, M. 1990. "Experimenting with Style in Archaeology: Some Historical and Theoretical Issues". En The Uses of Style in Archaeology, editado por M. Conkey y C. Hastorf, pp: 5-17. Cambridge University Press, Cambridge

Dunnell, R. 1986. "Five Decades of American Archaeology". En American Archaeology, Past and Future, editado por D. Meltzer, D. Fowler y J. Sabloff, pp: 23-49. Smithsonian Institution Press, Washington y Londres.

Lanning, E. 1967. Peru before the Incas. Prentice-Hall Inc., Englewood Cliffs, New Jersey.

Kroeber, A. 1927. "Coast and Highland in Prehistoric Peru". American Antiquity 29: 625-653.

Kroeber, A. 1944. Peruvian Archaeology in 1942. Viking Fund Publications in Anthropology No. 4, New York.

Lumbreras, L. 1984 "La Cerámica como indicador de culturas". Gaceta Arqueológica Andina 12: 3. 
Lothrop, S. y J. Mahler. 1957. Late Nazca burials in Chaviña, Peru. Papers of the Peabody Museum of Archaeology and Ethnology 50 (1). Harvard University, Cambridge, MA.

Massey, S. 1986. ociopolitical change in the upper Ica Valley, B. C. 400 to 400 A.D.: Regional States on the South Coast of Peru. Ph. D. Dissertation, Department of Anthropology, University of Los Angeles, Los Angeles.

Menzel, D. y F. Riddell. 1986. Archaeological Investigations at Tambo Viejo, Acari Valley, Peru, 1954. California Institute for Peruvian Studies, Sacramento.

Menzel, D., J. Rowe y L. Dawson. 1964. The Paracas Pottery from Ica: a Study of Style and Time. University of California Publications in American Archaeology and Ethnology. University of California Press, Berkeley y Los Angeles.

Orefici, O. y A. Drusini. 2003 Nasca: hipótesis y evidencias de su desarrollo cultural. Centro Italiano Studi e Ricerche Archaeologiche Precolombiane, Brescia.

Plog, S. 1990. "Sociopolitical Implications of Stylistic Variation in the American Southwest". En The Uses of Style in Archaeology, editado por M. Conkey y C. Hastorf, pp: 61-72. Cambridge University Press, Cambridge.

Proulx, D. 1968. Local Differences and Time Differences in Nasca Pottery. University of California Publications in Anthropology 5, Berkeley \& Los Angeles.

Proulx, D. 1971. "Headhunting in ancient Peru". Archaeology 24:16-21.

Proulx, D. 1989. "Nasca trophy heads: victims of warfare or ritual sacrifice?”. En Cultures in Conflict, editado por D. Tkaczuk y B. Vivian, pp: 7385. The Archaeological Association, University of Calgary, Calgary.

Proulx, D. 2001. "Ritual uses of trophy heads in ancient Nasca society". En Ritual Sacrifice in Ancient Peru., editado por E. Benson y A. Cook, pp: 119-136. University of Texas Press, Austin.

Rowe, J. 1960. "Cultural Unity and Diversification in Peruvian Archaeology". En Men and Cultures: Selected Papers of the Fifth International Congress of Anthropological and Ethnological Sciences, editado por A. Wallace, pp: 627-631. University of Pennsylvania Press, Philadelphia.

Rowe, J. 1956. "Archaeological explorations in Southern Peru, 1954-1955”. American Antiquity 22(2): 135-151. 
Rowe, J. 1963. "Urban settlements in ancient Peru”. Nawpa Pacha 1: 127.

Sackett, J. 1985. "Style, Ethnicity, and Stone Tools". En Status, Structure, and Stratification: Current Archaeological Reconstructions, editado por M. Thompson, M. García y F. Kense, pp: 277-282. The Archaeological Association, University of Calgary, Calgary.

Schreiber, K. 1999. "Regional approaches to the study of prehistoric empires: examples from Ayacucho and Nasca, Peru". En Settlement Pattern Studies in the Americas: Fifty Years since Virú, editado por B. Billman y G. Feinman, pp: 160-171. Smithsonian Institution Press, Washington, D.C

Silverman, H. 1988. "Cahuachi: non-urban cultural complexity on the South Coast of Peru”. Journal of Field Archaeology 15: 403-430.

Silverman, H. 1993. Cahuachi in the Ancient Nasca World. University of Iowa Press, Iowa City.

Silverman, H. 2002. Ancient Nasca Settlement and Society. University of Iowa Press, Iowa City.

Silverman, H. y D. Proulx. 2002. The Nasca. Blackwell Publishers, Malden, MA.

Strong, W. D. 1948. "Cultural Epochs and Refuse Stratigraphy in Peruvian Archaeology". En Reappraisal of Peruvian Archaeology, editado por W. Bennett, pp: 93-104. Memoirs of the Society for American Archaeology 4.

Valdez, L. 1994. "Cahuachi: New Evidence for an Early Nasca Ceremonial Role". Current Anthropology 35 (5): 675-679.

Valdez, L. 1998. The Nasca and the Valley of Acari: Cultural Interaction on the Peruvian South Coast Region during the First Four Centuries A.D. Ph.D. Dissertation, Department of Archaeology. University of Calgary, Calgary.

Valdez, L. 2000. "La Tradición Cultural Huarato de Acarí y sus Relaciones con Nasca”. Arqueología y Sociedad 13:159-171.

Valdez, L. 2005a."Patrones funerarios del periodo Intermedio Temprano del valle de Acarí". Corriente Arqueológica 1:43-60.

Valdez, L. 2005b. "Early Intermediate Period mortuary practices in the Acari Valley, Peru". Ponencia presentada al 45th. Annual Meeting of 
the Institute of Andean Studies, Enero 2005, University of California, Berkeley

Valdez, L. 2005c. Investigaciones Arqueológicas en Huarato, Acarí. Informe de la temporada del 2004 del Proyecto Arqueológico Acarí presentado al Instituto Nacional de Cultura, Lima.

Valdez, L. M. 2006. "Los vecinos de Nasca: entierros de la tradición Huarato del valle de Acarí, Perú". Bulletin de l'Institut Français d'Études Andines 35 (1): 1-20.

Valdez, L. M. 2007. "La investigación arqueológica en el valle de Acarí y la contribución de Francis A. Riddell, En Arqueología del Área Centro Sur Andina: Actas del Simposio Internacional. Andes 7:11-27. Boletín del Centro de Estudios Precolombinos de la Universidad de Varsovia.

Vaughn, K. 2000. Archaeological Investigations at Marcaya: a village approach to Nasca sociopolitical and economic organization. Ph. D. Dissertation, Department of Anthropology. University of California, Santa Barbara

Willey, G. 1948. "A Functional Analysis of "Horizon" Styles in Peruvian Archaeology”. En Reappraisal of Peruvian Archaeology, editado por W. Bennett, pp: 8-15. Memoirs of the Society for American Archaeology 4.

Willey, G. y J. Sabloff. 1980. A History of American Archaeology. W. H. Freeman and Company, New York 